\title{
The relationship between tumor budding and survival in colorectal carcinomas
}

1. Abant İzet Baysal University Hospital, Department of Pathology, Bolu, Turkey. 2. Haseki Training and Research Hospital, Department of Pathology, İstanbul, Turkey. 3. Abant İzet Baysal University Hospital, Department of General Surgery, Bolu, Turkey.

\section{SUMMARY}

OBJECTIVE: Tumor budding is a parameter that is increasingly understood in colorectal carcinomas. We aimed to investigate the relationship between tumor budding, prognostic factors, and survival.

METHODS: A total of 185 patients who had undergone colorectal surgery were observed. Tumor budding, the tumor budding score, and the relationship between these and prognostic factors, and survival investigated.

RESULTS: Tumor budding was found in 91 (49.2\%) cases. The relationship between the tumor budding score and histological grade, lymphovascular invasion, perineural invasion, pathological lymph node stage, and mortality rates were significant.

CONCLUSION: In our study, the relationship between tumor budding and survival is very strong. Considering these findings and the literature, the prognostic significance of tumor budding becomes clear and should be stated in pathology reports.

KEYWORDS: Colorectal Neoplasms. Survival. Neoplasm Staging.

\section{INTRODUCTION}

It is known that the pathological stage detected at the time of diagnosis in colorectal carcinoma (CRC) is the most important factor in determining the behavior and clinical course of tumors ${ }^{1}$. Recent studies have shown that patients at the same stage can show different prognosis, and, thus, new prognostic factors are being investigated, such as tumor budding. Tumor budding is thought to be a histological reflection of epithelial-mesenchymal transition (EMT). Cancer cells lose their epithelial properties such as polarity and adhesion during EMT; with the mesenchymal feature, they gain migratory capacity and become more resistant to apoptotic signals. The tumor cells that acquire these properties begin to separate, individually or in small groups, from the main mass ${ }^{2}$. Tumor budding was first described by Imai in 1954 as a morphological feature on the invasive front of the tumor, called tumor sprouting ${ }^{3}$. In 2002, Ueno et al. described tumor budding as a tumor cell or tumor-cell clusters of up to five cells, isolated from the main tumor on the invasive 
front. In the International Tumor Budding Consensus Conference (ITBCC), which was held on April 2016 with participants from 11 different countries, tumor budding was defined as a single tumor cell or a cell cluster of up to 4 tumor cells ${ }^{4}$.

Numerous studies have demonstrated that tumor budding is an independent prognostic factor associated with lymph node metastasis, local recurrence, and survival. The European Society for Medical Oncology (ESMO) and ITBCC guidelines included tumor budding as a criterion for identifying high-risk patient groups $^{4-6}$.

In the present study, we aimed to investigate the presence of tumor budding and the relationship between tumor budding and prognostic factors and survival in patients with colorectal carcinoma.

\section{METHODS}

A total of 240 patients who had undergone colorectal surgery at the Haseki Training and Research Hospital in Istanbul, Turkey, between 2008-2010 were observed. Fifty-five patients were excluded from the study. Among those, 20 patients received neo-adjuvant therapy, the slides of 20 patients could not be found, 14 patients died within a month, and one patient could not be reached for survival information. Colon and rectum resection materials of 185 patients were evaluated retrospectively. All histomorphologic data was reviewed based on the corresponding hematoxylin and eosin (H\&E) stained slides, whereas clinical data, tumor localization, tumor size, and surgical margins were obtained from corresponding reports. The survival information of the patients was observed from the records of the hospital information-record system and also from the patients by contacting them.

The H \& E stained preparations were re-evaluated for the presence of tumor budding, tumor budding score, tumor type, grade, invasion depth, lymphovascular invasion, perineural invasion, regional lymph node involvement, $\mathrm{pT}$, $\mathrm{pN}$ stages. Tumors were grouped as ascending colon, transverse colon, descending colon, sigmoid colon, and rectum according to their location.

We evaluated the histological subtypes following the World Health Organization (WHO) classification. Tumor invasion depth and lymph node evaluation were performed according to the $8^{\text {th }}$ edition of the American Cancer Committee (AJCC) tumor-nod-metastasis (TNM) classification. The presence and score of tumor budding were assessed based on the College of American Pathologists' (CAP) colorectal carcinoma reporting protocol. Accordingly, isolated tumor cells separated from the main tumor mass on the invasive front of the tumor or tumor cell clusters of up to 5 cells were classified as tumor budding?

Tumor buds were counted on the invasive margin at an area of $0.785 \mathrm{~mm}^{2}$. First, the cases were grouped as 'positive tumor budding' or 'negative tumor budding'. Then, positive tumor budding cases were scored according to the number of tumor buds. Cases with 0-4 tumor buds were scored as low grade, cases with 5-9 as moderate grade, and cases with $\geq 10$ as high grade $^{4,7}$. Routine immunohistochemical examination was not performed according to CAP protocol and ITBCC recommendations. Again, according to the CAP protocol and the recommendations of the ITBCC, an immunohistochemical examination was performed in 30 cases because of the inflammatory reaction surrounding the tumor, which was masking the tumor buds and the tumoral gland destruction by inflammatory cells, which simulates the tumor buds. For these cases, paraffin-embedded tissue blocks were cut at 2.5 $\mu \mathrm{m}$ thickness and immunostained for pan-cytokeratin (panCK), a marker of epithelial cells that served to highlight areas of tumor budding.

SPSS 15.0 for Windows was used for statistical analysis. The ratios were compared by chi-square analysis, and the relationships between ratios were analyzed with Linear-by-Linear Association. The relationships between numerical and ordinal variables were analyzed by Spearman Correlation Analysis. As the numerical variables did not meet the normal distribution condition, the two groups were compared with the Mann Whitney U test. Statistical significance was accepted as $\mathrm{p}<0.05$.

\section{RESULTS}

A total of 185 cases were included in the study. Of these, 107 were male, and 78 were female. The mean age of the patients was 60.5 years. Tumors were localized at the ascending colon in 59 cases (31.9\%), transverse colon in 11 cases (5.9\%), descending colon in 27 cases $(14.6 \%)$, sigmoid colon in 47 cases $(25.4 \%)$, and at the rectum in 41 cases $(22.2 \%)$. The tumor diameter was $6.1 \mathrm{~cm}$. The histological subgroup was classic adenocarcinoma in 170 (91.9\%) cases and mucinous adenocarcinoma in 15 (8.1\%) cases. The histologic grade was low in $30(16.2 \%)$ cases, moderate in 124 
(67\%, and high in 31 (16.8\%). Lymphovascular invasion was detected in 110 (59.5\%) cases and perineural invasion in $60(32.6 \%)$ cases. A total of 2 cases (1.1\%) were evaluated as pT1, 22 cases (11.9\%) as pT2, 125 (67.6\%) as pT3, and $36(19.5 \%)$ as pT4. The number of lymph nodes was between $0-44$, and the average number was 14. The lymph node involvement stage was NO in 99 (53.5\%) cases, N1a in $23(12.4 \%)$ cases, N1b in 29 (15\%), N2a in 19 (10.3\%), and N2b in 15 (8.1\%) cases. The mean follow-up period was 68.7 months and ranged from 2 to 108 months. A total of 104 cases (56.2\%) were alive, and 81 (43.8\%) died.

Tumor budding was found in 91 (49.2\%) cases; 49 cases $(26.5 \%)$ with low grade, $17(9.2 \%)$ with moderate, and 25 (13.5\%) with high-grade tumor budding.

No significant difference was observed between tumor budding and sex, age, tumor localization, tumor size, histological type of tumor, histological grade of the tumor, or pathologic T stage ( $p>0.05)$. Tumor budding was detected in 63 (69.2\%) of 110 cases with lymphovascular invasion and $39(42.9 \%)$ of 60 cases with perineural invasion. Both were significant $(p=0.008$ $\mathrm{p}=0.003$ ), (Table 1).

There were significant differences between the cases with and without tumor budding in terms of pathologic lymph node stages $(p=0.026)$. In addition, the rate of patients with tumor budding was lower than those without metastatic lymph nodes $(p=0.0049)$.
Also, the relationship between tumor budding and cumulative survival was significant; 48 (52.7\%) of the 91 cases with tumor budding died, and 43 (47.3\%) were still alive. The mortality rate of patients with tumor budding was significant $(p=0.016)$, as shown in Table 1.

The relationship between the tumor budding score and histological grade, lymphovascular invasion, perineural invasion, pathological lymph node stage and mortality rates were significant $(p=0.007, p<0.001$, $\mathrm{p}<0.001, \mathrm{p}=0.002, \mathrm{p}=0.001$ ). As the tumor budding scores increased, lymphovascular invasion, perineural invasion, $\mathrm{pN}$, and mortality rates increased too. As the tumor budding score increased, the rates of those with histological grade 1 decreased (Table 2).

The tumor budding score was found to be positively correlated with the number of metastatic lymph nodes, amd negatively correlated with the follow-up times $(p=0.011 p=0.001)$

The cumulative survival rate of patients with tumor budding was significantly lower than of those without tumor budding $(p=0.023)$ (Table 3).

There was a significant difference in the survival rates of tumor budding scores $(\mathrm{p}<0.001)$. The survival rates of patients with high tumor budding were significantly lower than those without tumor budding, or with low and moderate tumor budding scores. ( $p$ $<0.001 \mathrm{p}<0.001 \mathrm{p}=0.021)($ Table 3$)$.

TABLE 1. DISTRIBUTION OF TUMOR BUDDING BY LYMPHOVASCULAR AND PERINEURAL INVASION AND BY PN AND SURVIVAL INFORMATION

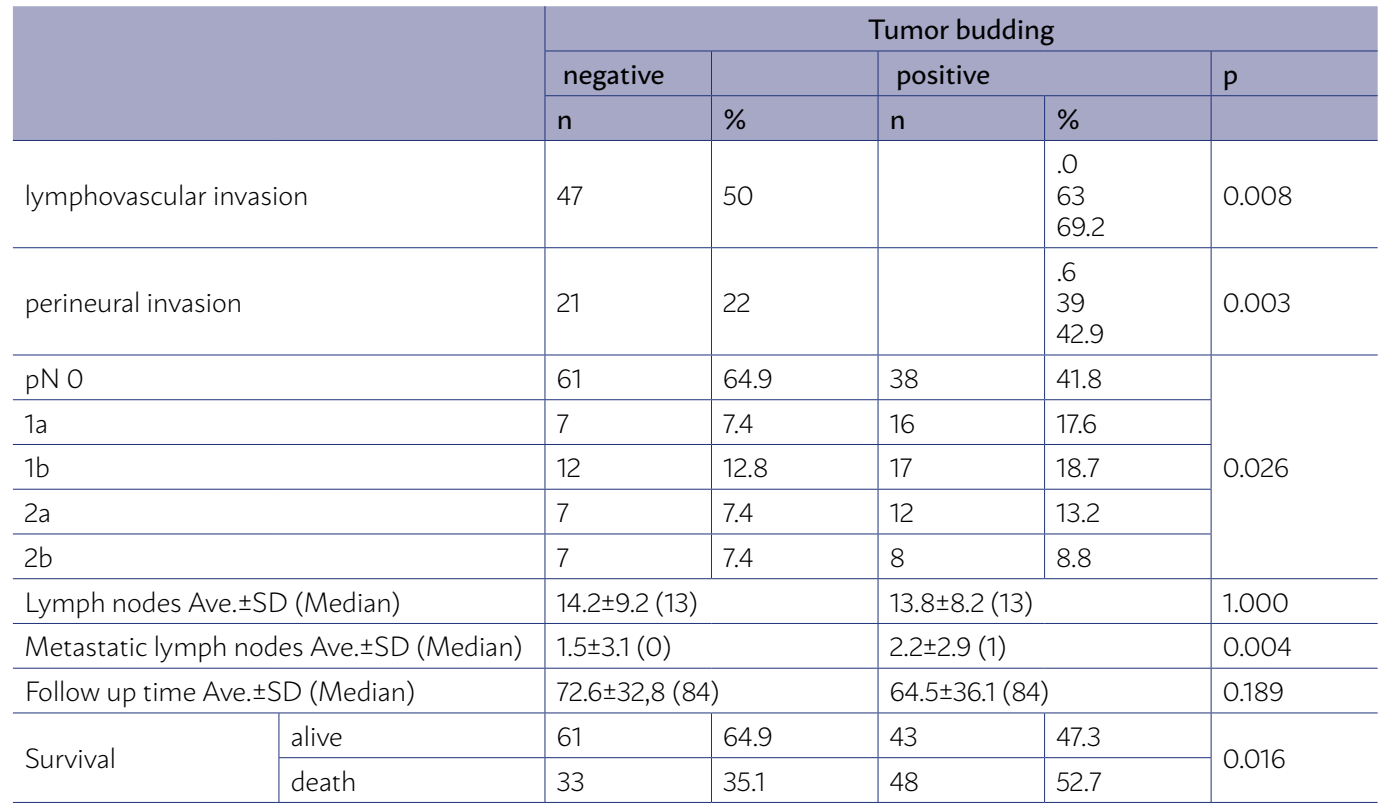




\section{DISCUSSION}

The number of colorectal carcinoma cases ranks $3^{\text {rd }}$ worldwide. Every year, 1.4 million people are diagnosed with CRC, and more than 600 thousand people lose their lives due to the disease ${ }^{8,9}$. After 50 years of age, it increases significantly, reaching the highest incidence in the second half of the eighth decade ${ }^{10}$.

The most important independent prognostic factor in CRCs is the stage of the tumor ${ }^{1}$. However, the fact that patients at the same pathological stage in the postoperative period present differences in terms of local recurrence and invasion suggests that pathological staging (TNM) is insufficient in these patients ${ }^{11}$. The situation required the investigation of biological, molecular, and morphological factors that may be related to the aggressive behavior of the tumor in the cancer tissue. Recent studies have focused on tumor budding, which is thought to be the first step of the metastatic process, among these morphological factors ${ }^{12-14}$.

In the literature, there is no significant relationship between tumor budding, age, sex and localization, diameter, or histologic subtype of tumor; we did not find any significant relationship either, ${ }^{5,12,15-21}$.

The relationship between tumor budding and the degree of histological differentiation of the tumor was not significant. However, the relationship between the tumor budding score and the degree of histological differentiation revealed a significant decrease in the rate of good differentiation. The relationship of tumor budding with pathological $\mathrm{T}$ stages has been investigated in many studies, and it was found that tumor budding is associated with advanced stages ${ }^{2,15-18,21-23}$. Koelzer et al. ${ }^{18}$ showed no significant relationship with pT. In our study, we found more tumor budding in pT3 stage, but this was not significant.

Few studies have demonstrated a significant correlation between tumor budding and perineural invasion $^{18,23,24}$. In our study, a significant correlation was found between the presence of tumor budding and the score of tumor budding and perineural invasion.

A significant relationship between tumor budding and lymphovascular invasion has been shown in most studies 2,15-18,21,23,24. In our study, the relationship between tumor budding and lymphovascular invasion

TABLE 2. THE RELATIONSHIP BETWEEN THE TUMOR BUDDING SCORE AND CLINICOPATHOLOGICAL PARAMETERS

\begin{tabular}{|c|c|c|c|c|c|c|c|c|c|c|}
\hline & & \multicolumn{9}{|c|}{ Tumor budding } \\
\hline & & \multicolumn{2}{|c|}{ negative } & \multicolumn{2}{|c|}{ low } & \multicolumn{2}{|c|}{ moderate } & \multicolumn{3}{|c|}{ high } \\
\hline & & $n$ & $\%$ & $n$ & $\%$ & $\mathrm{n}$ & $\%$ & $n$ & $\%$ & $\mathrm{p}$ \\
\hline \multirow{3}{*}{ Histologic grade } & 1 & 20 & 21.3 & 8 & 16.3 & 1 & 5.9 & 1 & 4.0 & \multirow{3}{*}{0.007} \\
\hline & 2 & 59 & 62.8 & 36 & 73.5 & 14 & 82.4 & 15 & 60.0 & \\
\hline & 3 & 15 & 16.0 & 5 & 10.2 & 2 & 11.8 & 9 & 36.0 & \\
\hline \multicolumn{2}{|c|}{ lymphovascular invasion } & 47 & 50 & 27 & 55.1 & 13 & 76.5 & 23 & 92 & $<0.001$ \\
\hline \multicolumn{2}{|c|}{ perineural invasion } & 21 & 22.6 & 17 & 34.7 & 5 & 29.4 & 17 & 68 & $<0.001$ \\
\hline \multirow{5}{*}{$\mathrm{pN}$} & 0 & 61 & 64.9 & 27 & 55.1 & 4 & 23.5 & 7 & 28.0 & \multirow{5}{*}{0.002} \\
\hline & $1 a$ & 7 & 7.4 & 8 & 16.3 & 4 & 23.5 & 4 & 16.0 & \\
\hline & $1 b$ & 12 & 12.8 & 6 & 12.2 & 3 & 17.6 & 8 & 32.0 & \\
\hline & $2 \mathrm{a}$ & 7 & 7.4 & 5 & 10.2 & 3 & 17.6 & 4 & 16.0 & \\
\hline & $2 b$ & 7 & 7.4 & 3 & 6.1 & 3 & 17.6 & 2 & 8.0 & \\
\hline \multirow{2}{*}{ Survival } & alive & 61 & 64.9 & 28 & 57.1 & 9 & 52.9 & 6 & 240 & \multirow{2}{*}{0.001} \\
\hline & death & 33 & 35.1 & 21 & 42.9 & 8 & 47.1 & 19 & 76.0 & \\
\hline
\end{tabular}

TABLE 3. THE RELATIONSHIP BETWEEN TUMOR BUDDING AND SURVIVAL

\begin{tabular}{|c|c|c|c|c|c|c|}
\hline & & \multicolumn{4}{|c|}{ Cumulative survival(\%) } & \multirow[b]{2}{*}{ Log Rank p } \\
\hline & & 12 month & 36 month & 60 month & 100 month & \\
\hline \multirow{2}{*}{ Tumor budding } & negative & 95.7 & 89.4 & 70.2 & 64.0 & 0.023 \\
\hline & positive & 89.0 & 69.2 & 56.0 & 47.3 & \\
\hline \multirow{4}{*}{ Tumor budding score } & negative & 93.7 & 76.6 & 70.2 & 64.0 & \multirow{2}{*}{$<0.001$} \\
\hline & low & 93.9 & 79.6 & 67.3 & 58.3 & \\
\hline & moderate & 100.0 & 76.5 & 58.8 & 50.4 & \\
\hline & high & 72.0 & 44.0 & 32.0 & 24.0 & \\
\hline
\end{tabular}


was significant. In addition, as the degree of tumor budding increases, the rate of cases with lymphovascular invasion increases too, so there is a significant relationship between the tumor budding score and lymphovascular invasion.

Numerous studies have examined the relationship of tumor budding with lymph node metastasis. In 2012, Kye et al..$^{25}$ compared the relationship between regional lymph node metastasis and all other prognostic factors in 55 patients with stage pT1; among all parameters, only tumor budding was found to be an independent prognostic factor for lymph node metastasis. In our study, the relationship of tumor budding with metastatic lymph node number and lymph node stage was significant. In addition, the relationship between the tumor budding score and $\mathrm{pN}$ stage is significant; as the degree of tumor budding increases, the $\mathrm{pN}$ is also increased.

As the prognostic importance of tumor budding was understood, the number of studies investigating the relationship with survival also increased. All these studies have shown that the presence of tumor budding is associated with significant poor clinical outcomes and shorter survival time.

In the study by Ohtsuki et al. ${ }^{23}$, the disease-free survival rate was $40.9 \%$ for cases with tumor budding and $75.1 \%$ in cases without tumor budding. Rogers et al. ${ }^{26}$ reported that tumor budding was an independent prognostic factor related to cancer-related death in univariate and multivariate analyses. When we look at the relationship between tumor budding and cumulative survival, of the 91 cases with tumor budding, 48 (52.7\%) died, and 43 (47.3\%) were still alive. The mortality rate of patients with tumor budding was significant. There was a significant correlation between the tumor budding degrees and follow-up periods in patients with tumor budding. The cumulative survival rate of patients with tumor budding was significantly lower than those without tumor budding. In addition, the relationship between the score of tumor budding and cumulative survival is significant. The cumulative survival rate of patients with high tumor budding rates is significantly lower than other scores and no tumor budding.

\section{CONCLUSION}

In our study, the relationship between tumor budding and survival is very strong. Considering these findings and the literature, the prognostic significance of tumor budding becomes clear and should be stated in pathology reports.

\section{Authors contributions}

The idea for research or article/hypothesis generation: SB.Planning the methods to generate hypothesis: SP, SB.

Supervision and responsibility for the organization and course of the project and the manuscript preparation: SP, SB.

Supplying financial resources, equipment, space, and personnel vital to the Project: SP, SB, BÖ

Biological materials, reagents, referred patients: BÖ, OÇ, MŞ

\section{RESUMO}

OBJETIVO: Brotamento de tumor é um parâmetro que é cada vez mais conhecido em carcinomas colorretais. Nosso objetivo foi investigar a relação entre brotamento tumoral e fatores prognósticos e sobrevida.

MÉTODOS: Um total de 240 pacientes observados, submetidos à cirurgia colorretal. Brotamento de tumor, escore de brotamento tumoral e a relação entre estes e fatores prognósticos, sobrevida investigada.

RESULTADOS: Brotamento de tumores foi encontrado em 91 (49,2\%) casos. A relação entre o escore de brotamento tumoral e o grau histológico, invasão linfovascular, invasão perineural, estadiamento linfonodal patológico e taxas de mortalidade foram significativas.

CONCLUSÃo: Em nosso estudo, a relação entre brotamento tumoral e sobrevida é muito forte. Em conjunto, todos esses achados e literatura são avaliados simultaneamente, o significado prognóstico da brotação do tumor é claramente visto e deve ser indicado nos relatórios de patologia.

PALAVRAS-CHAVE: Neoplasias Colorretais. Sobrevida. Estadiamento de Neoplasias. 


\section{REFERENCES}

1. Amin MB, Greene FL, Edge SB, Compton CC, Gershenwald JE, Brookland RK, et al. The Eighth Edition AJCC Cancer Staging Manual: continuing to build a bridge from a population-based to a more "personalized" approach to cancer staging. CA Cancer J Clin. 2017;67(2):93-9.

2. Zlobec I, Lugli A. Epithelial mesenchymal transition and tumor budding in aggressive colorectal cancer: tumor budding as oncotarget. Oncotarget. 2010;1(7):651-61

3. Okuyama T, Oya M, Yamaguchi M. Budding (sprouting) as a useful prognostic marker in colorectal mucinous carcinoma. Jpn | Clin Oncol. 2002;32(10):412-6

4. Lugli A, Kirsch R, Ajioka Y, Bosman F, Cathomas G, Dawson H, et al. Recommendations for reporting tumor budding in colorectal cancer based on the International Tumor Budding Consensus Conference (ITBCC) 2016. Mod Pathol. 2017;30(9):1299-311.

5. Koelzer VH, Zlobec I, Lugli A. Tumor budding in colorectal cancer: ready for diagnostic practice? Hum Pathol. 2016;47(1):4-19.

6. Schmoll H, Van Cutsem E, Stein A, Valentini V, Glimelius B, Haustermans K, et al. ESMO Consensus Guidelines for management of patients with colon and rectal cancer. a personalized approach to clinical decision making. Ann Oncol. 2012;23(10):2479-516.

7. Cho SI, Kakar S. Tumor budding in colorectal carcinoma: translating a morphologic score into clinically meaningful results. Arch Pathol Lab Med. 2018;142(8):952-7.

8. Ferlay J, Soerjomataram I, Ervik M, Dikshit R, Eser S, Mathers C, et al. GLOBOCAN 2012: estimated cancer incidence, mortality and prevalence worldwide in 2012. [cited 2019 May 12]. Available from: https://publications. iarc.fr/Databases/larc-Cancerbases/GLOBOCAN-2012-Estimated-Cancer-Incidence-Mortality-And-Prevalence-Worldwide-In-2012-V1.0-2012

9. Torre LA, Bray F, Siegel RL, Ferlay J, Lortet-Tieulent J, Jemal A. Global cancer statistics, 2012. CA Cancer J Clin. 2015;65(2):87-108.

10. Richman SD, Southward K, Chambers P, Cross D, Barrett |, Hemmings G, et al. HER2 overexpression and amplification as a potential therapeutic target in colorectal cancer: analysis of 3256 patients enrolled in the QUASAR, FOCUS and PICCOLO colorectal cancer trials. | Pathol. 2016;238(4):562-70.

11. Park KJ, Choi HJ, Roh MS, Kwon HC, Kim C. Intensity of tumor budding and its prognostic implications in invasive colon carcinoma. Dis Colon Rectum. 2005;48(8):1597-602.

12. Ueno H, Murphy J, Jass IR, Mochizuki H, Talbot IC. Tumour 'budding' as an index to estimate the potential of aggressiveness in rectal cancer. Histopathology. 2002;40(2):127-32

13. Nakamura T, Mitomi H, Kikuchi S, Ohtani Y, Sato K. Evaluation of the usefulness of tumor budding on the prediction of metastasis to the lung and liver after curative excision of colorectal cancer. Hepatogastroenterology. 2005;52(65):1432-5.
14. Ueno H, Mochizuki H, Hashiguchi $Y$, Shimazaki H, Aida S, Hase K, et al. Risk factors for an adverse outcome in early invasive colorectal carcinoma. Gastroenterology. 2004;127(2):385-94.

15. Graham RP, Vierkant RA, Tillmans LS, Wang AH, Laird PW, Weisenberger D), et al. Tumor budding in colorectal carcinoma: confirmation of prognostic significance and histologic cutoff in a population-based cohort. Am | Surg Pathol. 2015;39(10):1340-6.

16. Kazama S, Watanabe T, Ajioka Y, Kanazawa T, Nagawa H. Tumour budding at the deepest invasive margin correlates with lymph node metastasis in submucosal colorectal cancer detected by anticytokeratin antibody CAM5. 2. Br J Cancer. 2006;94(2):293-8.

17. Satoh K, Nimura S, Aoki M, Hamasaki M, Koga K, Iwasaki H, et al. Tumor budding in colorectal carcinoma assessed by cytokeratin immunostaining and budding areas: possible involvement of c-Met. Cancer Sci. 2014:105(11):1487-95

18. Koelzer VH, Assarzadegan N, Dawson H, Mitrovic B, Grin A, Messenge $\mathrm{DE}$, et al. Cytokeratin-based assessment of tumour budding in colorectal cancer: analysis in stage II patients and prospective diagnostic experience. | Pathol Clin Res. 2017;3(3):171-8.

19. Horcic M, Koelzer VH, Karamitopoulou E, Terracciano L, Puppa G, Zlobec I, et al. Tumor budding score based on 10 high-power fields is a promising basis for a standardized prognostic scoring system in stage II colorectal cancer. Hum Pathol. 2013;44(5):697-705.

20. Nakamura T, Mitomi H, Kanazawa H, Ohkura Y, Watanabe M. Tumor budding as an index to identify high-risk patients with stage II colon cancer. Dis Colon Rectum. 2008;51(5):568-72.

21. Karamitopoulou E, Zlobec I, Kölzer V, Kondi-Pafiti A, Patsouris ES, Gennatas K, et al. Proposal for a 10-high-power-fields scoring method for the assessment of tumor budding in colorectal cancer. Mod Pathol. 2013;26(2):295-301.

22. Zlobec I, Hädrich M, Dawson H, Koelzer V, Borner M, Mallaev M, et al. Intratumoural budding (ITB) in preoperative biopsies predicts the presence of lymph node and distant metastases in colon and rectal cancer patients. Br J Cancer. 2014;110(4):1008-13.

23. Ohtsuki K, Koyama F, Tamura T, Enomoto Y, Fujii H, Mukogawa T, et al. Prognostic value of immunohistochemical analysis of tumor budding in colorectal carcinoma. Anticancer Res. 2008;28(3B):1831-6.

24. Wang LM, Kevans D, Mulcahy H, O'Sullivan J, Fennelly D, Hyland J, et al. Tumor budding is a strong and reproducible prognostic marker in T3NO colorectal cancer. Am J Surg Pathol. 2009;33(1):134-41.

25. Kye BH, Jung JH, Kim HJ, Kang SG, Cho HM, Kim JG. Tumor budding as a risk factor of lymph node metastasis in submucosal invasive T1 colorectal carcinoma: a retrospective study. BMC Surg. 2012;12:16.

26. Rogers AC, Gibbons D, Hanly AM, Hyland JM, O'Connell PR, Winter DC, et al. Prognostic significance of tumor budding in rectal cancer biopsies before neoadjuvant therapy. Mod Pathol. 2014;27(1):156-62. 